\title{
COMPLETELY ZERO-SIMPLE SEMIGROUPS GENERATED BY NILPOTENT ELEMENTS
}

\author{
by C. H. HOUGHTON and R. P. SULLIVAN
}

(Received 15 December, 1982)

For a completely 0-simple semigroup, Howie [2] has investigated the subsemigroup generated by the idempotents. Here we determine those elements of such a semigroup which are generated by the set of nilpotent elements and hence we derive a condition for a completely 0 -simple semigroup to be nilpotent generated. This condition is purely combinatorial, in terms of the structure of the graph associated with the semigroup, and it includes the case of a non-regular Rees matrix semigroup.

1. The combinatorial interpretation. The Rees matrix semigroup $M=$ $\mathcal{M}^{0}(G ; I, \Lambda ; P)$ is formed from a group $G$, disjoint sets $I$ and $\Lambda$, and a $\Lambda \times I$ matrix $P=\left(p_{\lambda i}\right)$ with entries in $G^{0}$. The non-zero elements of $M$ are the triples $g_{i \lambda}$ with multiplication $\left(g_{i \lambda}\right)\left(h_{j \mu}\right)=\left(g \cdot p_{\lambda j} \cdot h\right)_{i \mu}$. An element $g_{i \lambda}$ is idempotent if and only if $p_{\lambda i} \neq 0$ and $g=p_{\lambda i}^{-1}$. Thus the set $E$ of idempotents is bijective with $\Gamma=\left\{(\lambda, i): p_{\lambda i} \neq 0\right\}$, which we regard as the edge set of a bipartite graph with vertex set $\Lambda \cup I$. The graph may be defined abstractly as having $E$ as its set of directed edges, with $e, f \in E$ having common source if $e f=e$ and $f e=f$ or having common target if $e f=f$ and $f e=e$. Thus the graph is an isomorphism invariant of $M$.

A semigroup is completely 0 -simple if and only if it is isomorphic to a regular Rees matrix semigroup. The condition for $M$ to be regular is that the matrix $P$ has a non-zero entry in each row and column. In terms of graphs, this means that no vertex of $\Gamma$ is isolated.

The graph $\Gamma$ is complete if $\Gamma=\Lambda \times I$ and this occurs when the semigroup is the union of 0 and a completely simple semigroup. Then 0 is the only nilpotent element and the nilpotent generated subsemigroup is $\{0\}$. For general $\Gamma$, we define the adjacency sets of vertices by $\operatorname{adj}(\lambda)=\{i:(\lambda, i) \in \Gamma\}$ and $\operatorname{adj}(i)=\{\lambda:(\lambda, i) \in \Gamma\}$.

The definition of multiplication shows that $g_{i \lambda} \in M$ is nilpotent if and only if $p_{\lambda i}=0$ or, equivalently, $(\lambda, i) \notin \Gamma$; it follows that $g_{i \lambda}^{2}=0$. Writing $(i, g, \lambda)$ for $g_{i \lambda}$, a product $\left(i_{1}, g_{1}, \lambda_{1}\right)$ $\left(i_{2}, g_{2}, \lambda_{2}\right) \ldots\left(i_{n}, g_{n}, \lambda_{n}\right)$ will be non-zero if and only if $\left(\lambda_{1}, i_{2}\right),\left(\lambda_{2}, i_{3}\right), \ldots,\left(\lambda_{n-1}, i_{n}\right) \in \Gamma$. Thus an element $g_{i \lambda}$ is a product of nilpotents if and only if there is a sequence $i=i_{1}, \lambda_{1}, i_{2}, \lambda_{2}, \ldots, i_{n}, \lambda_{n}=\lambda$ with $\left(\lambda_{j}, i_{j}\right) \notin \Gamma$ and $\left(\lambda_{j}, i_{j+1}\right) \in \Gamma$, that is, a sequence alternately of non-edges and edges from $i$ to $\lambda$, the last term being a non-edge.

An element $s=g_{i \lambda}$ will be called a 0 -divisor if there is an element $t \neq 0$ with $s t=0$ or $t s=0$; this occurs if and only if $\operatorname{adj}(\lambda) \neq I$ or $\operatorname{adj}(i) \neq \Lambda$. We remark that the conditions for $g_{\mathrm{i} \lambda}$ to be nilpotent, nilpotent generated, or a 0 -divisor depend only on properties of $\Gamma$ and are independent of the value of $g$. Our first result is straightforward.

Glasgow Math. J. 25 (1984) 163-165. 
THEOREM 1. Let $S$ be a completely 0-simple semigroup.

(a) If the associated graph $\Gamma$ is disconnected then $S$ is nilpotent generated.

(b) If $S$ is orthodox then it is nilpotent generated unless it is a completely simple semigroup with 0 adjoined.

Proof. (a) Given $i$ and $\lambda$ with $(\lambda, i) \notin \Gamma$ then all $g_{i \lambda}$ are nilpotent. If $(\lambda, i) \in \Gamma$ then, since $\Gamma$ is disconnected and no vertex is isolated, there is an edge $(\mu, j)$ in a different component. The sequence $i, \mu, j, \lambda$ shows that $g_{i \lambda}$ is a product of two nilpotents.

(b) From Corollary 5.2 of [1], an orthodox semigroup has more than one component in its graph, except in the specified case.

So far all the nilpotent generated elements that we have exhibited have been products of at most two nilpotents. We give an example showing that, in general, products of arbitrary length may necessarily arise.

For $n \geqq 3$ let $\Lambda=\{1,2, \ldots, n\}, I=\{n+1, n+2, \ldots, 2 n\}$ and define $\Gamma$ by adjacency sets as follows:

$$
\begin{aligned}
\operatorname{adj}(1) & =\{n+1, n+2, \ldots, 2 n-1\}, \\
\operatorname{adj}(2) & =\{n+2\}, \\
\operatorname{adj}(3) & =\{n+1, n+3\}, \\
\operatorname{adj}(4) & =\{n+1, n+2, n+4\}, \\
\operatorname{adj}(5) & =\{n+1, n+2, n+3, n+5\}, \\
\operatorname{adj}(n) & =\{n+1, n+2, \ldots, 2 n-2,2 n\} .
\end{aligned}
$$

Then the shortest possible sequence of non-edges and edges from $n+1$ to $n$ is $n+1$, $2, n+2,3, n+3,4, \ldots, n-1,2 n-1, n$ and so $g_{n+1, n}$ is the product of $n-1$ nilpotents, with no fewer sufficing. We remark that the edge $(n, n+1)$ turns our sequence into a cycle of alternately non-edges and edges visiting each vertex and so a semigroup with this graph is nilpotent generated.

2. Characterisation. We now give a general method for constructing completely 0 -simple semigroups which are not nilpotent generated and we shall show that any such semigroup has the form of our construction.

THeorem 2. Let $A, B, C, D$ be disjoint sets, put $\Lambda=A \cup B, I=C \cup D$, and take $\Gamma \subseteq \Lambda \times I$ with $B \times C \subseteq \Gamma$ and $\Gamma \cap(A \times D)=\varnothing$. If $M$ is a corresponding Rees matrix semigroup with group $G$ then $g_{i \lambda}$ is nilpotent if $(\lambda, i) \in A \times D$, but $g_{i \lambda}$ is not nilpotent generated if $(\lambda, i) \in B \times C$. For $(\lambda, i) \in A \times C$ or $B \times D$, the corresponding properties are controlled by the subgraphs $\Gamma \cap(A \times C)$ and $\Gamma \cap(B \times D)$ respectively.

Proof. For $M$ to exist, $\Lambda \neq \varnothing \neq I$. Since $\Gamma \cap(A \times D)=\varnothing$, all $g_{\text {ii }}$ with $(\lambda, i) \in A \times D$ are nilpotent. If $i \in C$ and there is a sequence of non-edges and edges from $i$ to $\lambda \in \Lambda$ then all the terms $(\mu, j)$ lie in $A \times C$ and, in particular, $\lambda \in A$. This shows that $g_{i \lambda}$ is not a product of nilpotents for $(\lambda, i) \in B \times C$ and also demonstrates the reduction for $(\lambda, i) \in$ $A \times C$. A similar argument holds for $B \times D$. 
By taking $A, B, C$ and $D$ non-empty and requiring no vertex to be isolated we obtain completely 0 -simple semigroups which are not nilpotent generated although all elements are 0-divisors; the smallest such $\Gamma$ has $|A|=|D|=|B|=|C|=2$. Since $\Gamma \cap(A \times C)$ and $\Gamma \cap(B \times D)$ are arbitrary, any bipartite graph without isolated vertices may arise as a subgraph of a $\Gamma$ associated with a completely 0 -simple semigroup.

THEOREM 3. Let $\Gamma$ be the graph associated with a Rees matrix semigroup which is not nilpotent generated. Then $\Gamma$ has the structure described in Theorem 2 with $B \neq \varnothing \neq C$.

Proof. Since the semigroup has non-nilpotent elements, $\Gamma$ is non-empty and we may choose $(\lambda, i) \in \Gamma$. If $\Lambda \times\{i\} \subseteq \Gamma$ then we have the required structure with $A=\varnothing, B=\Lambda$, $C=\{i\}, D=I \backslash\{i\}$. Otherwise take $A$ to be the set of all $\mu \in \Lambda$ reached from $i$ by a sequence of non-edges and edges. Then $A$ is non-empty and, since $\lambda \notin A$, so also is $B=\Lambda \backslash A$. If $C=\operatorname{adj}(A)$ and $D=I \backslash C$ then $\Gamma \cap(A \times D)=\varnothing$. For $(\mu, j) \in B \times C$, we have $(\nu, j) \in \Gamma$, for some $\nu \in A$, and then there is a sequence of non-edges and edges from $i$ to $\nu$ to $j$. Now $\mu \notin A$ and so we must have $(\mu, j) \in \Gamma$. Thus $B \times C \subseteq \Gamma$.

Given $\Gamma$ as in Theorems 2 and 3 , suppose that there exists $\lambda \in A$ with $\operatorname{adj}(\lambda)=C$. If we replace $B$ by $B \cup\{\lambda\}$ and $A$ by $A \backslash\{\lambda\}$ then the required properties are preserved. We may similarly enlarge $C$ to contain all $i$ with $\operatorname{adj}(i)=B$. Thus in our decomposition we may assume that $B \times C$ is a maximal complete subgraph. Conversely, to decide if a graph has the given structure we need only examine its maximal complete subgraphs. We note that repeated application of Theorems 2 and 3 gives an effective procedure for determining the nilpotent generated elements; in practice, a systematic investigation of sequences of non-edges and edges is usually quicker.

\section{REFERENCES}

1. C. H. Houghton, Completely 0 -simple semigroups and their associated graphs and groups, Semigroup Forum 14 (1977), 41-67. 109-113.

2. J. M. Howie, Idempotents in completely 0-simple semigroups, Glasgow Math. J. 19 (1978),

Department of Pure Mathematics

University COLLEGE

CARDIFF

DePaRTMENT OF MATHEMATICS

UNIVERSTTY OF WESTERN AUSTRALIA 